\title{
Correlation between Health Literacy and Quality of Life in Iranian Menopausal Women
}

\author{
Ensiyeh Jenabi ${ }^{1}$, Behzad Gholamaliee ${ }^{2}$, Salman Khazaei ${ }^{3}$ \\ ${ }^{1}$ Autism Spectrum Disorders Research Center, Hamadan University of Medical Sciences, Hamadan, Iran, ${ }^{2}$ School of Public Health, \\ Hamadan University of Medical Sciences, Hamadan, Iran, ${ }^{3}$ Research Center for Health Sciences, Hamadan University of Medical \\ Sciences, Hamadan, Iran
}

Objectives: Understanding the factors that promote quality of life $(\mathrm{OOL})$ in menopausal women is essential for establishing educational strategies and health policies. Studies on the relationship between health literacy and $00 \mathrm{~L}$ in menopausal women in Iran are rare. Therefore, we aimed to determine health literacy in menopausal women and its relationship with QOL.

Methods: The present cross-sectional study was performed on 177 menopausal women referred to comprehensive health services in Hamadan City, Iran. Health Literacy for Iranian Adults and Menopause-Specific QOL questionnaires were used to collect information. Pearson correlation coefficient was used to assess the correlation between health literacy and 00L.

Results: The majority of menopausal women included in this study were married housewives, and $44.6 \%$ of them were $50-55$ years old; furthermore, only $6.2 \%$ had adequate or good health literacy. Health literacy was significantly correlated with total 00L score and vasomotor, psychology, and physical domains $(P<0.05)$.

Conclusions: Health literacy was associated with various domains of $\mathrm{QOL}$. Therefore, health providers should pay more attention on improving health literacy by holding educational promotional programs for menopausal women to enhance their QOL.

Key Words: Health literacy, Iran, Menopause, Quality of life

\section{INTRODUCTION}

Menopause is resulting from the permanent cessation of menstruation, which occurs around the age of 51 in women [1]. Due to increase in the human life expectancy in recent years, nearly one third of women's life is located after menopause [2]. In Iran like other developing countries the number of middle aged women has raised dramatically in recent years. Several factors contribute to the quality of life (QOL) in postmenopausal women. Physiological changes during menopause, various mental problems such as depression and memory loss, as well as sexual dysfunctions, reduced physical activity and engage in underlying diseases such as hypertension, diabetes type 2 , osteoporosis, and muscu- loskeletal diseases can reduce dramatically their QOL $[1,3]$.

The QOL is an important end point in medicine and researches on disease outcomes [4]. QOL indicated that how individuals intuitively evaluate their health as well as their ability to perform physical, psychological, and social functions [5-7]. Currently, QOL is measured for judge about outcome as a comprehensive health indicator in medical interventions and health surveys [8].

The health of human societies is affected by many factors such as economic development, social and cultural factors. One of them is health literacy, which is a term introduced by the American Medical Association in $1970[8,9]$. Health literacy is defines as "a constellation of skills, including the ability to perform basic reading

Received: December 1, 2019 Revised: March 2, 2020 Accepted: March 3, 2020

Address for Correspondence: Salman Khazaei, Research Center for Health Sciences, Hamadan University of Medical Sciences, Hamedan, Hamadan Province 65178, Iran

Tel: +98-38380496, E-mail: salman.khazaei61@gmail.com, ORCID: https://orcid.org/0000-0001-5918-2310 
and numerical tasks required to function in the health care environment" [8].

In modern societies, literacy and health literacy are considered as essential components of health and poor health literacy has adverse effects on peopless health [10]. Evidence shows that some vulnerable groups such as elderly people, immigrants, low level of education, societies with low level of socio-economic status, people with mental health problem, and people with chronic diseases have low levels of health literacy [11].

Knowing the factors relating promoting of QOL in menopausal women is essential for educational strategies and health policy making. Evidence regarding the evaluation of the health literacy and its relationship with QOL in menopausal women in Iran is rare [12], therefore in this study we aimed to determine health literacy in menopausal women and its relationship with QOL.

\section{MATERIALS AND METHODS}

The present study was a cross-sectional study performed on 177 menopausal women referred to comprehensive health services of Hamadan City, Iran. This study was conducted for duration of 3 consecutive months in 2019. Sampling method was quota random selection in comprehensive health services of Hamadan City. According to study of Abdolmalaki et al. [13], sample size was estimated 190 menopausal woman based on power $80 \%$ and $\alpha=5 \%$ with a $10 \%$ dropout rate.

Inclusion criteria was menopausal women aged between 40-60 years referred to comprehensive health services of Hamadan City, not having history of physical and mental disorders and not receiving hormone replacement therapy. Exclusion criteria were failure to fulfill the questionnaire correctly and completely. The study protocol was reviewed and approved by Ethical Committee of Hamadan University of Medical Sciences (ethical code: IR.UMSHA.REC.1398.416). All participants filled out and signed informed consent form.

In total, three questionnaires were used in this study. The first questionnaire was including demographic characteristics, such as age, education level, marital status, and job.

Health Literacy for Iranian Adults (HELIA) was the second questionnaire with alpha coefficient correlation score ranging from $0.72-0.89$ which included 33 questions (in the dimensions of availability, reading, under- standing, assessment and decision-making and use of health information). The validity and reliability of this questionnaire been confirmed by Montazeri et al. [14]. Each question has a 5-point scale including "it is quite easy", "it is easy", "it is hard", "it is quite hard", and "not easy not hard", respectively. According to the questionnaire, menopausal women were divided into four different categories: well (84.1-100), adequate (66.1-84), partially adequate (50.1-66), and inadequate (less than $50)$.

The third questionnaire was Menopause-Specific QOL (MENQOL) questionnaire. This questionnaire measure QOL in postmenopausal women includes of 29 items in vasomotor (3 questions), psychosocial (7 questions), physical (16 questions), and sexual (3 questions) dimensions [15]. The validity and reliability for this questionnaire were conducted in Iran [16]. This questionnaire has seven-point Likert scale with ranges between $0-7$. Score zero is equivalent to a woman responding "no", reporting women has not experienced this symptom in the past month. Score "one" reports that she experienced the symptom, but it was not bothersome at all. Scores "two" until "seven" report increasing levels of bother experienced from the symptom and correspond to " 1 " through " 6 " check boxes on the

Table 1. Basic charecreristics of included women

\begin{tabular}{lc}
\hline \multicolumn{1}{c}{ Charecreristic } & Number (\%) \\
\hline Age $(\mathrm{y})$ & $1(0.6)$ \\
$40-45$ & $60(33.9)$ \\
$45-50$ & $79(44.6)$ \\
$50-55$ & $37(20.9)$ \\
$55-60$ & \\
Education ${ }^{\mathrm{a}}$ & $27(15.3)$ \\
Illiterate & $63(35.8)$ \\
Elementary & $80(45.5)$ \\
High school & $6(3.4)$ \\
University & \\
Marital status & $151(85.3)$ \\
Married & $26(14.7)$ \\
Divorse or widow or unmarried & \\
Job & $168(94.9)$ \\
Housewife & $9(5.1)$ \\
Employed & \\
\hline
\end{tabular}

${ }^{\mathrm{a} D}$ Data of 1 person was missed. 
MENQOL. Each item was manually calculated into a 0-7 score. Hence, the average for each domain was calculated between 0 and 7. The high scores in MENQOL dimensions indicate low QOL.

We used IBM SPSS ver. 20 (IBM Corp., Armonk, NY, USA) was in order to analyzing the data. The Pearson correlation coefficient was used. The statistically significant was considered with $P$ values lower than 0.05 and two-tailed hypotheses.

\section{RESULTS}

Rate of the response to questions was $93 \%$. A total of 13 women did not fully complete the questionnaires and were deleted from the present study. Therefore, 177 menopausal women contributed in this study.

Table 2. Results of MENQOL and HELIA

\begin{tabular}{lr}
\multicolumn{1}{c}{ Questionnaire } & \multicolumn{1}{c}{ Score } \\
\hline MENQOL dimensions & \\
Vasomotor & $6.70 \pm 3.90$ \\
Psychosocial & $13.45 \pm 7.75$ \\
Physical & $27.31 \pm 1.35$ \\
Sexual dimensions & $4.42 \pm 3.67$ \\
HELIA dimensions & \\
Availability & $18.51 \pm 5.54$ \\
Reading & $12.43 \pm 4.13$ \\
Understanding & $22.39 \pm 7.90$ \\
Assessment & $10.27 \pm 4.40$ \\
Decision-making and use of health information & $40.39 \pm 9.98$ \\
\hline
\end{tabular}

Values are presented as mean \pm standard deviation.

MENQOL: Menopause-Specific quality of life, HELIA: Health Literacy for Iranian Adults.
The mean age of menopausal women was $51.30 \pm 3.65$ years. Most of menopausal women were housewife (94.9\%) and married (85.3\%). Also, 45.5\% had education level of high school. The mean of menarche age was 12.28 years and temporal relationship between marriage and childbirth was 1.43 years. Table 1 presents the characteristics of the menopausal women in present study. The mean scores of QOL for vasomotor, psychosocial, physical and sexual dimensions were $6.70 \pm 3.90$, $13.45 \pm 7.75,27.31 \pm 1.35$, and $4.42 \pm 3.67$, respectively. Also, the mean scores of HELIA for availability, reading, understanding, assessment, and decision-making and use of health information dimensions were $18.51 \pm$ $5.54,12.43 \pm 4.13,22.39 \pm 7.90,10.27 \pm 4.40$, and $40.39 \pm$ 9.98, respectively (Table 2).

The results of Table 3 showed that $46.3 \%$ had health literacy of inadequate, $47.5 \%$ had health literacy of partially adequate, $5.6 \%$ had health literacy of adequate, and $0.6 \%$ had health literacy of well.

The results of the Table 4 showed that the score mean of availability dimension of HELIA questionnaire was significantly correlated with the mean scores of vasomotor, psychosocial, physical, sexual, and mean score of MENQOL in postmenopausal women $(P<0.05)$. There was a significant relationship between assessment dimension of HELIA and mean score of vasomotor, psychosocial, sexual, and total mean score of MEN-

Table 3. Frequency distribution of health literacy in the participants

\begin{tabular}{lc}
\hline Health literacy & Number $(\%)$ \\
\hline Inadequate & $82(46.3)$ \\
Partially adequate & $84(47.5)$ \\
Adequate & $10(5.6)$ \\
Well & $1(0.6)$ \\
\hline
\end{tabular}

Table 4. The correlation between health literacy with quality of life in menopausal women

\begin{tabular}{|c|c|c|c|c|c|}
\hline \multirow{2}{*}{ HELIA dimension } & \multicolumn{4}{|c|}{ MENQOL dimension } & \multirow{2}{*}{$\begin{array}{c}\text { Total of scores } \\
\text { MENQOL }\end{array}$} \\
\hline & Vasomotor & Psychology & Physical & Sexual & \\
\hline Availability & $-0.292^{\mathrm{a}}$ & $-0.324^{\mathrm{a}}$ & $-0.181^{\mathrm{a}}$ & -0.081 & $-0.261^{\mathrm{a}}$ \\
\hline Reading & 0.061 & 0.139 & 0.04 & 0.301 & 0.108 \\
\hline Understanding & 0.142 & 0.107 & 0.009 & 0.102 & 0.066 \\
\hline Assessment & $-0.214^{\mathrm{a}}$ & $-0.209^{\mathrm{a}}$ & -0.073 & $-0.165^{\mathrm{a}}$ & $-0.165^{\mathrm{a}}$ \\
\hline Decision-making and use of health information & $-0.277^{\mathrm{a}}$ & $-0.322^{\mathrm{a}}$ & -0.132 & -0.045 & $-0.225^{\mathrm{a}}$ \\
\hline Total of scores health literacy & $-0.326^{\mathrm{a}}$ & $-0.373^{\mathrm{a}}$ & $-0.209^{\mathrm{a}}$ & 0.001 & $-0.285^{\mathrm{a}}$ \\
\hline
\end{tabular}

HELIA: Health Literacy for Iranian Adults, MENQOL: Menopause-Specific quality of life.

${ }^{\text {a }}$ Significant at $5 \%$ level. 
QOL in postmenopausal women $(P<0.05)$. There was a significant relationship between decision-making, and the use of health information dimension of HELIA questionnaire with mean score of vasomotor, psychosocial, and total mean score of MENQOL. There was a significant relationship between total mean score of HELIA questionnaire in postmenopausal women with mean score of vasomotor, psychosocial, physical, and total mean score of MENQOL $(P<0.05)$.

\section{DISCUSSION}

The results showed that only $6.2 \%$ of menopause women had adequate or well health literacy. Health literacy was significantly in correlation with total score of QOL, vasomotor, psychology, and physical domains. It means that QOL decreases as health literacy decreases and vice versa.

The positive relation between adequate level of health literacy with the QOL has been approved previously in research subjects in Iran [12] and other countries $[17,18]$. Although, Zhang et al. [18], in their study reached a contradict result. This difference can be due to the different applied questionnaires to measure of health literacy and QOL in their study populations. Also differences in culture, ethnicity and social impacts of the subjects are among the factors affecting the QOL and health literacy of subjects and confounded this association [19].

Abdolmalaki et al. [13] in 2018 showed that $47.8 \%$ menopausal women had inadequate health literacy. In our study, $46.3 \%$ menopausal women had inadequate health literacy. Therefore, this study was in line with our findings.

Improving health literacy is associated with behavioral changes and playing active role in decision making inadequate health literacy is associated with non-compliance to the physician orders, inappropriate drugs consumption, poor control of weight, blood glucose, blood lipid and blood pressure, low health knowledge, poor communication between physician and patient and consequently decreased QAL [20]. Yadollahi et al. [21], showed that improving health literacy is associated with behavioral changes and playing active role in decision making. Therefore not wonder that World Health Organization considered health literacy a major determining factor for health [20]. Despite the importance of health literacy in promoting health in present study, less than $10 \%$ of participations had adequate, although this situation in exist in other parts of Iran and even in developed countries [22,23].

In current study, there were some limitations: First, socioeconomic status is an important factor which can effect on QOL of postmenopausal women, while we studied a group with only $3.4 \%$ had academic degree and majority were housewife, therefore assessing health literacy and QOL may be encountered with underestimation. Second, information about QOL of the participants during their premenopausal period was based on self-reporting and therefore prone to information bias. Further studies with stronger design like cohort or case-control and larger sample size is recommended to better understanding the association.

In conclusion, we found that postmenopausal women in Hamadan province had low level of health literacy and given that health literacy has an impact on the various domains of their QOL, therefore health providers should focus more attention on improving people's health literacy by holding educational promotion programs for menopausal women.

\section{ACKNOWLEDGMENTS}

The authors would like to appreciate the Vice-chancellor of Research and Technology, Hamadan University of Medical Sciences, Iran for financial support of the study (research ID: 9805223860).

\section{CONFLICT OF INTEREST}

No potential conflict of interest relevant to this article was reported.

\section{REFERENCES}

1. Greendale GA, Lee NP, Arriola ER. The menopause. Lancet 1999; 353: 571-80.

2. Nisar N, Sohoo NA. Severity of menopausal symptoms and the quality of life at different status of menopause: a community based survey from rural Sindh, Pakistan. Int J Collab Res Intern Med Public Health 2010; 2: 118-30

3. de Souza Santos CA, Dantas EE, Moreira MH. Correlation of physical aptitude; functional capacity, corporal balance and quality of life (QoL) among elderly women submitted to a post-menopausal physical activities program. Arch Gerontol Geriatr 2011; 53: 344-9.

4. Welton AJ, Vickers MR, Kim J, Ford D, Lawton BA, MacLennan $\mathrm{AH}$, et al. Health related quality of life after combined hormone 
replacement therapy: randomised controlled trial. BMJ 2008; 337: a1190.

5. Wang HM, Beyer M, Gensichen J, Gerlach FM. Health-related quality of life among general practice patients with differing chronic diseases in Germany: cross sectional survey. BMC Public Health 2008; 8: 246.

6. Shobeiri F, Jenabi E, Hazavehei SM, Roshanaei G. Quality of life in postmenopausal women in Iran: a population-based study. J Menopausal Med 2016; 22: 31-8.

7. Jenabi E, Shobeiri F, Hazavehei SM, Roshanaei G. Assessment of questionnaire measuring quality of life in menopausal women: a systematic review. Oman Med J 2015; 30: 151-6.

8. Miguel RS, López-González AM, Sanchez-Iriso E, Mar J, Cabasés JM. Measuring health-related quality of life in drug clinical trials: is it given due importance? Pharm World Sci 2008; 30: 154-60.

9. Sørensen K, Van den Broucke S, Fullam J, Doyle G, Pelikan J, Slonska Z, et al. Health literacy and public health: a systematic review and integration of definitions and models. BMC Public Health 2012; 12: 80 .

10. Kickbusch I, Pelikan JM, Apfe F, Tsouros AD. Health literacy: the solid facts. Copenhagen: World Health Organization Regional Office for Europe; 2013.

11. Jabalameli S, Doost HTN, Moulavi H. Efficacy of cognitivebehavioral stress management intervention on quality of life and blood pressure in female patients with hypertension. Sci J Kurd Univ Med Sci 2010; 15: 88-97.

12. Mosallanezhad Z, Poornowrooz N, Javadpour S, Haghbeen M, Jamali S. Health literacy and its relationship with quality of life in postmenopausal women. J Clin Diagn Res 2019; 13: VC01-04.

13. Abdolmalaki B, Peyman N, Esmaeili H. Evaluation of health literacy rate of postmenopausal women covered by Mashhad health centers in 2018. J Health Lit 2018; 3: 182-90.
14. Montazeri A, Tavousi M, Rakhshani F, Azin SA, Jahangiri K, Ebadi $\mathrm{M}$, et al. Health literacy for Iranian adults (HELIA): development and psychometric properties. Payesh 2014; 13: 589-99.

15. Lewis JE, Hilditch JR, Wong CJ. Further psychometric property development of the menopause-specific quality of life questionnaire and development of a modified version, MENQOL-intervention questionnaire. Maturitas 2005; 50: 209-21.

16. Fallahzadeh H. Quality of life after the menopause in Iran: a population study. Qual Life Res 2010; 19: 813-9.

17. Shin KM, Chu SH, Jang YS, Kang SM. Relationships between health literacy, knowledge and self-care behaviors in elderly patients with heart failure. J Korean Clin Nurs Res 2016; 22: 28-37.

18. Zhang XH, Li SC, Fong KY, Thumboo J. The impact of health literacy on health-related quality of life (HRQoL) and utility assessment among patients with rheumatic diseases. Value Health 2009; 12 Suppl 3: S106-9.

19. Chew LD, Bradley KA, Boyko EJ. Brief questions to identify patients with inadequate health literacy. Fam Med 2004; 36: 588-94.

20. Javadzade SH, Sharifirad G, Radjati F, Mostafavi F, Reisi M, Hasanzade A. Relationship between health literacy, health status, and healthy behaviors among older adults in Isfahan, Iran. J Educ Health Promot 2012; 1: 31.

21. Yadollahi M, Siavashi E, Mostaghim S. The relationship between health literacy and patient participation in medical decision making among breast cancer patients. Arch Breast Cancer 2018; 5 : 183-8.

22. Yee LM, Simon MA. The role of health literacy and numeracy in contraceptive decision-making for urban Chicago women. J Community Health 2014; 39: 394-9.

23. Tavousi M, Haeri MA, Rafiefar S, Solimanian A, Sarbandi F, Ardestani $\mathrm{M}$, et al. Health literacy in Iran: findings from a national study. Payesh 2016; 15: 95-102. 